\title{
LIMIT MEASURES RELATED TO THE CONDITIONALLY FREE CONVOLUTION
}

\author{
MELANIE HINZ \\ Institut für Mathematik und Informatik, Ernst-Moritz-Arndt Universität Greifswald, \\ Jahnstrasse 15a, D-17487 Greifswald, Germany \\ melanie.hinz@uni-greifswald.de \\ WOJCIECH MŁOTKOWSKI \\ Institute of Mathematics, Wrocław University, \\ Pl. Grunwaldzki 2/4, 50-384 Wrocław, Poland \\ mlotkow@math.uni.wroc.pl
}

January 2, 2007

\begin{abstract}
We describe the limit measures for some class of deformations of the free convolution, introduced by A. D. Krystek and E. J. Wojakowski.
\end{abstract}

Keywords: Cauchy transform; conditionally free product; Jacobi coefficients. AMS Subject Classification: 46L54, 42C05, 60E10.

\section{Introduction}

The conditionally free convolution, defined by Bożejko, Leinert and Speicher[1], is an associative and commutative operation $\boxplus$ on pairs of compactly supported probability measures on $\mathbb{R}$. It is closely related to the Voiculescu free convolution, namely, if

$$
\left(\mu_{1}, \nu_{1}\right) \boxplus\left(\mu_{2}, \nu_{2}\right)=(\mu, \nu)
$$

then $\nu=\nu_{1} \boxplus \nu_{2}$, and if $\mu_{1}=\nu_{1}$ and $\mu_{1}=\nu_{2}$ then $\mu=\mu_{1} \boxplus \mu_{2}$. 
Recall that an important tool for studying a probability measure $\mu$ on $\mathbb{R}$ is the Cauchy transform, the analytic function $G_{\mu}: \mathbb{C}_{+} \rightarrow \mathbb{C}$ defined by

$$
G_{\mu}(z):=\int_{\mathbb{R}} \frac{d \mu(t)}{z-t}
$$

where $\mathbb{C}_{+}:=\{z \in \mathbb{C}: \operatorname{Im} z>0\}$. If $\mu$ is compactly supported then $G_{\mu}(z)$ can be represented as a continued fraction

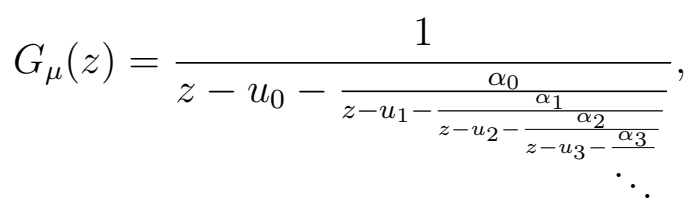

for which we will use more convenient notation:

$$
G_{\mu}(z)=\frac{1 \mid}{\mid z-u_{0}}-\frac{\alpha_{0} \mid}{\mid z-u_{1}}-\frac{\alpha_{1} \mid}{\mid z-u_{2}}-\frac{\alpha_{2} \mid}{\mid z-u_{3}}-\frac{\alpha_{3} \mid}{\mid \ldots} .
$$

The Jacobi coefficients satisfy: $\alpha_{k} \geq 0, u_{k} \in \mathbb{R}$ and if $\alpha_{m}=0$ for some $m \geq 0$ then $\alpha_{n}=u_{n}=0$ for all $n>m$. The coefficient $\alpha_{0}$ is called the variance of $\mu$ and denoted by $V(\mu)$. We will need the following two properties, which can be found in the Chihara monograph[2].

Proposition 1.1 Assume that $\mu$ is a compactly supported probability measure on $\mathbb{R}$, with the Cauchy transform given by (2). Then:

(i) $\mu$ is symmetric (i.e. $\mu(A)=\mu(-A)$ holds for every Borel subset of $\mathbb{R}$ ) if and only if $u_{k}=0$ for every $k \geq 0$.

(ii) The support of $\mu$ is contained in the halfline $[0,+\infty)$ if and only if there exists a sequence $\left\{\lambda_{m}\right\}_{m \geq 0}$ of nonnegative numbers such that for every $m \geq 0$ we have $\alpha_{m}=\lambda_{2 m} \cdot \lambda_{2 m+1}$ and $u_{m}=\lambda_{2 m}+\lambda_{2 m-1}$, under convention that $\lambda_{-1}=0$.

The numbers $\lambda_{m}$ are the nonnegative (i.e. the upper) Jacobi coefficients for the symmetric measure $\mu^{\text {sym }}$ defined by: $\int_{\mathbb{R}} f\left(t^{2}\right) d \mu^{\mathrm{sym}}(t)=\int_{\mathbb{R}} f(t) d \mu(t)$.

To define the conditionally free convolution we define two transforms. For a pair $\mu, \nu \in \mathcal{M}_{c}$ we define $R_{\nu}, R_{\mu, \nu}$ as the complex functions which satisfy

$$
\begin{aligned}
& \frac{1}{G_{\nu}(z)}=z-R_{\nu}\left(G_{\nu}(z)\right), \\
& \frac{1}{G_{\mu}(z)}=z-R_{\mu, \nu}\left(G_{\nu}(z)\right)
\end{aligned}
$$


(the former is the Voiculescu free transform). For $\mu_{1}, \nu_{1}, \mu_{2}, \nu_{2} \in \mathcal{M}_{c}$ the conditionally free convolution (1) is defined by

$$
\begin{gathered}
R_{\nu}(z)=R_{\nu_{1}}(z)+R_{\nu_{2}}(z), \\
R_{\mu, \nu}(z)=R_{\mu_{1}, \nu_{1}}(z)+R_{\mu_{2}, \nu_{2}}(z) .
\end{gathered}
$$

Now, assume that $T$ is a map on the class $\mathcal{M}_{c}$ of compactly supported probability measures on $\mathbb{R}$, which satisfies the following condition (called Bożejko property): if

$$
\left(\mu_{1}, T \mu_{1}\right) \boxplus\left(\mu_{2}, T \mu_{2}\right)=(\mu, \nu)
$$

then $\nu=T \mu$. Defining $\mu_{1} \boxplus_{T} \mu_{2}:=\mu$ we obtain an associative and commutative operation $\boxplus_{T}$ on $\mathcal{M}_{c}$. For example, if $T \mu=\mu$ for every $\mu \in \mathcal{M}_{c}$ then $\boxplus_{T}$ is the Voiculescu free convolution $\boxplus$, and if $T \mu=\delta_{0}$ for every $\mu \in \mathcal{M}_{c}$ then $\boxplus_{T}$ becomes the Boolean convolution $\uplus$.

\section{The $\phi$-convolution}

From now on we fix a compactly supported probability measure $\phi$ on $\mathbb{R}$ which is infinitely divisible with respect to $\boxplus$. Let

$$
G_{\phi}(z)=\frac{1 \mid}{\mid z-\beta_{0}}-\frac{\gamma_{0} \mid}{\mid z-\beta_{1}}-\frac{\gamma_{1} \mid}{\mid z-\beta_{2}}-\frac{\gamma_{2} \mid}{\mid z-\beta_{3}}-\frac{\gamma_{3} \mid}{\mid \ldots}
$$

be its Cauchy transform. Krystek and Wojakowski [3] defined a convolution $\boxplus_{\phi}$ in the following way. For $\mu \in \mathcal{M}_{c}$, we put $T \mu:=\phi^{\boxplus V(\mu)}$ (the free power of $\phi)$. Then $T$ admits Bożejko property (Theorem 7 in Ref. [3]) and we denote $\boxplus_{\phi}:=\boxplus_{T}$. The authors of Ref.[3] found the related limit measures only in the case when $\phi$ is either the Wigner or the free Poisson measure.

We are going to exhibit the relation between the Jacobi coefficients of $\phi$ and those of the limit measures with respect to $\boxplus_{\phi}$. In particular we prove that a modified version of hypothesis given in Ref. [3] Remark 10 is true.

For $\mu \in \mathcal{M}_{c}$ and $\lambda>0$ we define dilation of $\mu$ by $\mathcal{D}_{\lambda} \mu(A):=\mu\left(\lambda^{-1} A\right)$. Denote by $\gamma_{m}^{(\lambda)}, \beta_{m}^{(\lambda)}$ the Jacobi coefficients of the free power $\phi^{\boxplus \lambda}$.

Theorem 2.1 (The central limit theorem) Assume that $\mu \in \mathcal{M}_{c}$ satisfies $\int_{\mathbb{R}} t d \mu(t)=0$ and $\int_{\mathbb{R}} t^{2} d \mu(t)=\lambda$. Then the sequence

$$
\xi_{\lambda, N}:=\mathcal{D}_{\frac{1}{\sqrt{N}}} \mu \boxplus_{\phi} \cdots \boxplus_{\phi} \mathcal{D}_{\frac{1}{\sqrt{N}}} \mu,
$$


$N$ times, is $*$-weakly convergent to the measure $\xi_{\lambda} \in \mathcal{M}_{c}$ such that

$$
G_{\xi_{\lambda}}(z)=\frac{1 \mid}{\mid z}-\frac{\lambda \mid}{\mid z-\beta_{0}^{(\lambda)}}-\frac{\gamma_{0}^{(\lambda)} \mid}{\mid z-\beta_{1}^{(\lambda)}}-\frac{\gamma_{1}^{(\lambda)} \mid}{\mid z-\beta_{2}^{(\lambda)}}-\frac{\gamma_{2}^{(\lambda)} \mid}{\mid \ldots}
$$

In particular, $\xi$ is symmetric if and only if $\phi$ is symmetric.

Proof. By (slightly generalized version of) Ref. [3], Theorem 8 and (4) we have $\frac{1}{G_{\xi_{\lambda}}(z)}=z-\lambda G_{\phi^{\boxplus \lambda}}(z)$, which proves (5). It remains to use Proposition $1(\mathrm{i})$.

Theorem 2.2 (The Poisson limit theorem) For $\lambda>$ the sequence

$$
\rho_{\lambda, N}:=\left(\left(1-\frac{\lambda}{N}\right) \delta_{0}+\frac{\lambda}{N} \delta_{1}\right) \boxplus_{\phi} \cdots \boxplus_{\phi}\left(\left(1-\frac{\lambda}{N}\right) \delta_{0}+\frac{\lambda}{N} \delta_{1}\right),
$$

$N$ times, is $*$-weakly convergent to the measure $\rho_{\lambda}$, which satisfies

$$
G_{\rho_{\lambda}}(z)=\frac{1}{\mid z-\lambda}-\frac{\lambda \mid}{\mid z-\beta_{0}^{(\lambda)}-1}-\frac{\gamma_{0}^{(\lambda)} \mid}{\mid z-\beta_{1}^{(\lambda)}}-\frac{\gamma_{1}^{(\lambda)} \mid}{\mid z-\beta_{2}^{(\lambda)}}-\frac{\gamma_{2}^{(\lambda)} \mid}{\mid \ldots}
$$

Moreover, the support of $\rho_{\lambda}$ is contained in $[0,+\infty)$ if and only if the support of $\phi$ is contained in $[0,+\infty)$.

Proof. According to Ref. [3], Theorem 9 and formula (4) we have

$$
\frac{1}{G_{\rho_{\lambda}}(z)}=z-\frac{\lambda}{1-G_{\phi^{\boxplus \lambda}}(z)}=z-\lambda-\frac{\lambda}{\frac{1}{G_{\phi^{\boxplus \lambda}}(z)}-1},
$$

which leads to (6).

Assume that $\operatorname{supp}(\phi) \subseteq[0,+\infty)$, then also $\operatorname{supp}\left(\phi^{\boxplus \lambda}\right) \subseteq[0,+\infty)$. Let $\left\{\lambda_{m}\right\}_{m=0}^{\infty}$ be the sequence of upper Jacobi coefficients of $\left(\phi^{\boxplus \lambda}\right)_{\text {sym }}$, according to Proposition 1.1(ii). Then the numbers $\lambda_{0}^{\prime}:=\lambda, \lambda_{1}^{\prime}:=1$ and $\lambda_{k}^{\prime}:=\lambda_{k-2}$ for $k \geq 2$, are the upper Jacobi coefficients for $\left(\rho_{\lambda}\right)_{\mathrm{sym}}$. On the other hand, if the support of $\rho_{\lambda}$ is contained in $[0, \infty)$ and if $\lambda_{m}^{\prime}$ are the upper Jacobi coefficients of $\left(\rho_{\lambda}\right)_{\text {sym }}$ then we have $\lambda_{0}^{\prime}=\lambda, \lambda_{1}^{\prime}=1$ and the numbers $\lambda_{m+2}$, $m \geq 0$, are the upper Jacobi coefficients of $\left(\phi^{\boxplus \lambda}\right)_{\mathrm{sym}}$.

Remark 2.1 The limit measures $\xi_{\lambda}$ and $\rho_{\lambda}$ are $\boxplus_{\phi}$-infinitely divisible. More generally, let $\mu=\mu(\lambda, u, v)$, with $\lambda>0, u, v \in \mathbb{R}$, denote such a measure $\mu$ that

$$
G_{\mu}(z)=\frac{1 \mid}{\mid z-u}-\frac{\lambda \mid}{\mid z-\beta_{0}^{(\lambda)}-v}-\frac{\gamma_{0}^{(\lambda)} \mid}{\mid z-\beta_{1}^{(\lambda)}}-\frac{\gamma_{1}^{(\lambda)} \mid}{\mid z-\beta_{2}^{(\lambda)}}-\frac{\gamma_{2}^{(\lambda)} \mid}{\mid \ldots} .
$$


Then we have $V(\mu)=\lambda$ and

$$
\frac{1}{G_{\mu}(z)}=z-u-\frac{\lambda}{\frac{1}{G_{\phi^{\boxplus \lambda}}(z)}-v}=z-u-\frac{\lambda G_{\phi^{\boxplus \lambda}}(z)}{1-v G_{\phi^{\boxplus \lambda}}(z)},
$$

which, by 4, leads

$$
R_{\mu, \phi^{\boxplus \lambda}}(z)=u+\frac{\lambda z}{1-v z}
$$

Therefore we have

$$
\mu\left(\lambda_{1}, u_{1}, v\right) \boxplus_{\phi} \mu\left(\lambda_{2}, u_{2}, v\right)=\mu\left(\lambda_{1}+\lambda_{2}, u_{1}+u_{2}, v\right)
$$

and for $t>0$

$$
\mu(\lambda, u, v)^{\boxplus_{\phi} t}=\mu(t \lambda, t u, v),
$$

which means that all the measures $\mu(\lambda, u, v)$ are $\boxplus_{\phi}$-infinitely divisible.

\section{Acknowledgment}

Research supported by RTN: HPRN-CT-2002-00279, W. Młotkowski is also sponsored by KBN: 1P03A 01330 and by ToK: MTKD-CT-2004-013389. This work was carried out partly during stay of Melanie Hinz in Wrocław (5.03-31.07.2006) and of Wojciech Młotkowski in Greifswald (26.10-5.11.2006).

\section{References}

[1] M. Bożejko, M. Leinert, R. Speicher, Convolution and limit theorems for conditionally free random variables, Pacific J. Math. 175 no. 2 (1996), $357-388$.

[2] T. S. Chihara, An Introduction to Orthogonal Polynomials, Gordon and Breach.

[3] A. D. Krystek, Ł. J. Wojakowski, Associative convolutions arising from conditionally free convolution, Infin. Dim. Anal. Quantum Probab. Related Top. 8 (2005), 515-545. 